UCRL-JC-121639

PREPRINT

\title{
CONF- $960163-23$
}

\section{Computational Modeling of Ultra-Short-Pulse Ablation of Enamel}

\author{
R. A. London, D. S. Bailey, D. A. Young, W. E. Alle \\ M. D. Feit, A. M. Rubenchik, and J. Neev

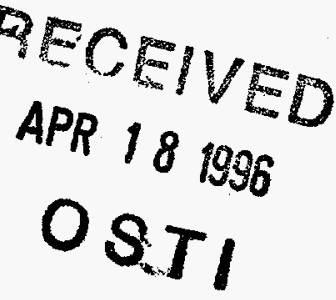

This paper was prepared for submittal to the SPIE Biomedical Optics Symposium '96

San Jose, CA

January 27-February 2, 1996

February 29, 1996

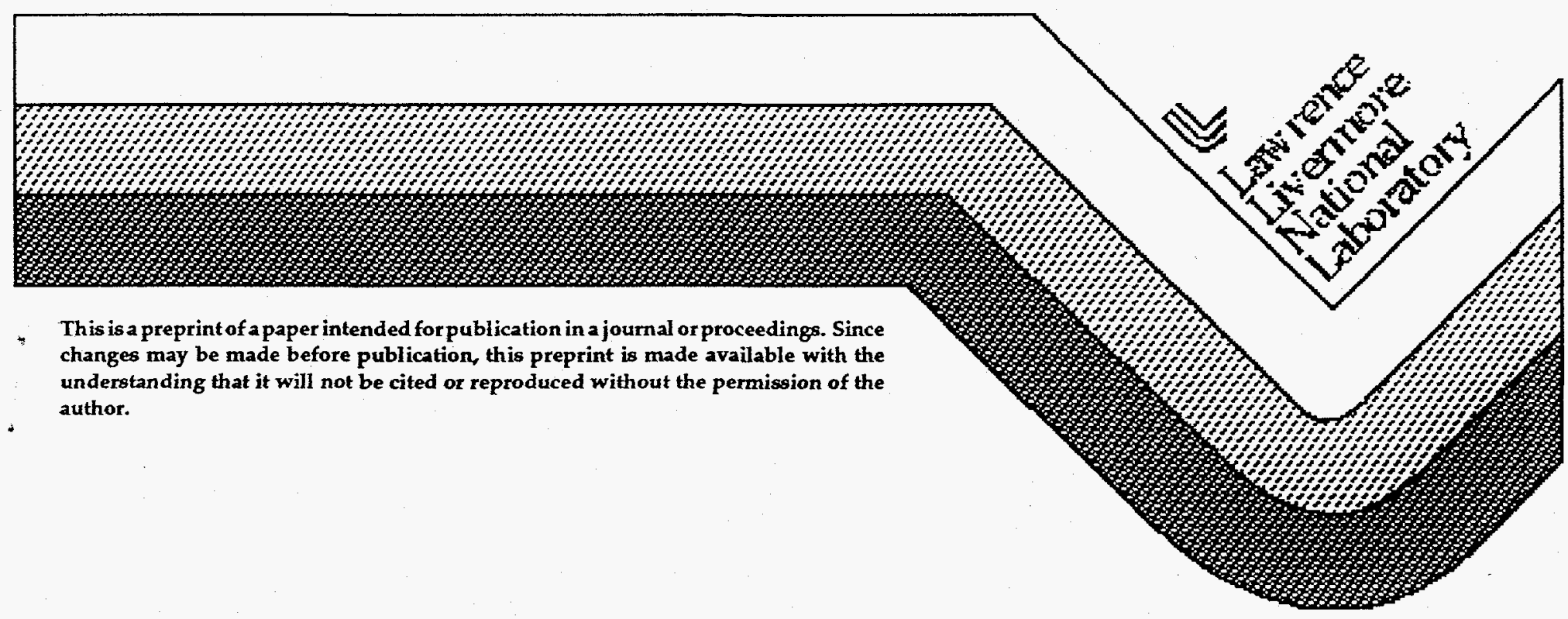




\section{DISCLAIMER}

This document was prepared as an account of work sponsored by an agency of the United States Government. Neither the United States Government nor the University of California nor any of their employees, makes any warranty, express or implied, or assumes any legal liability or responsibility for the accuracy, completeness, or usefulness of any information, apparatus, product, or process disclosed, or represents that its use would not infringe privately owned rights. Reference herein to any specific commercial product, process, or service by trade name, trademark, manufacturer, or otherwise, does not necessarily constitute or imply its endorsement, recommendation, or favoring by the United States Government or the University of California. The views and opinions of authors expressed herein do not necessarily state or reflect those of the United States Government or the University of California, and shall not be used for advertising or product endorsement purposes. 


\section{DISCLAMMER}

Portions of this document may be illegible in electronic image products. Images are produced from the best available original document. 


\title{
Computational Modeling of Ultra-Short-Pulse Ablation of Enamel
}

\author{
Lawrence Livermore National Laboratory \\ Livermore, California 94550 \\ and \\ J. Neev \\ Beckman Laser Institute \\ Irvine, California 92715
}

R. A. London, D. S. Bailey, D. A. Young, W. E. Alley, M. D. Feit, and A. M. Rubenchik

\begin{abstract}
A computational model for the ablation of tooth enamel by ultra-short laser pulses is presented. The role of simulations using this model in designing and understanding laser drilling systems is discussed. Pulses of duration $300 \mathrm{fsec}$ and intensity greater than $10^{12}$ $\mathrm{W} / \mathrm{cm}^{2}$ are considered. Laser absorption proceeds via multi-photon initiated plasma mechanism. The hydrodynamic response is calculated with a finite difference method, using an equation of state constructed from thermodynamic functions including electronic, ion motion, and chemical binding terms. Results for the ablation efficiency are presented. An analytic model describing the ablation threshold and ablation depth is presented. Thermal coupling to the remaining tissue and long-time thermal conduction are calculated. Simulation results are compared to experimental measurements of the ablation efficiency. Desired improvements in the model are presented.
\end{abstract}

Keywords: laser-tissue ablation, laser-tissue modeling, ultra-short-pulse lasers, dental ablation

\section{INTRODUCTION}

Ultra-short-pulse laser (USPL) ablation holds promise for high precision tissue removal with minimal collateral damage. Specifically we consider pulses in the fsec to psec range, which are focused to high intensities on the tissue $\left(>1012 \mathrm{~W} / \mathrm{cm}^{2}\right)$. In this regime, the dominant laser absorption mechanism is high density plasma absorption, initiated by multi-photon ionization. This very strong process leads to absorption coefficients in the range of 1 to $100 \mu \mathrm{m}^{-1}$. Because of the small absorption depth and short pulse duration (compared to thermal and hydrodynamic timescales), the absorbed energy is highly concentrated in a small volume; therefore the ablation threshold occurs at a relatively low fluence. Recent experiments have observed an ablation threshold $\approx$ $3 / 4 \mathrm{~J} / \mathrm{cm}^{2}$ for dental enamel ${ }^{1}$. Associated with the short length and time scales are relatively low thermal and mechanical coupling to the unablated tissue. Thus USPL ablation can be both precise and minimally damaging. In the context of hard dental tissues (enamel and dentin), an USPL can avoid the problems of thermal and mechanical damage which have plagued attempts to develop instruments based on 
traditional long pulse lasers. With the future development of relatively low cost, high repetition rate systems ${ }^{2}$, USPLs can compete in speed with standard dental drills, opening the door to widespread use. In addition to dental applications, USPLs may find a role in other hard-and soft-tissue removal medical procedures.

The specific aim of this paper is to demonstrate the role of numerical simulations in understanding and in designing an optimal system for dental applications.

The paper is laid out as follows: section 2 discusses the elements of the model and the computer code, section 3 gives hydrodynamic simulation results, section 4 presents an analytic theory describing the main hydro results, and section 5 gives results of heat diffusion simulations. Plans for future work and conclusions are presented in section 6 .

\section{FUNDAMENTALS OF THE NUMERICAL MODEL}

Time dependent simulations of the laser interaction and the reaction of the tissue are performed with the LATIS computer program 3,4 . LATIS is based on over 20 years of experience in modeling laser-matter interaction at high intensities in the laser-fusion program ${ }^{5}$. LATIS considers four primary processes in modeling laser-tissue interaction, as illustrated in Figure 1: laser propagation and thermal, hydrodynamic and material response. In previous work, we have concentrated on the simulations involving laser propagation, thermal response and microscopic thermal damage. In the present work we apply new capabilities involving the hydrodynamic response, and the material properties of equation-of-state and material strength and failure.

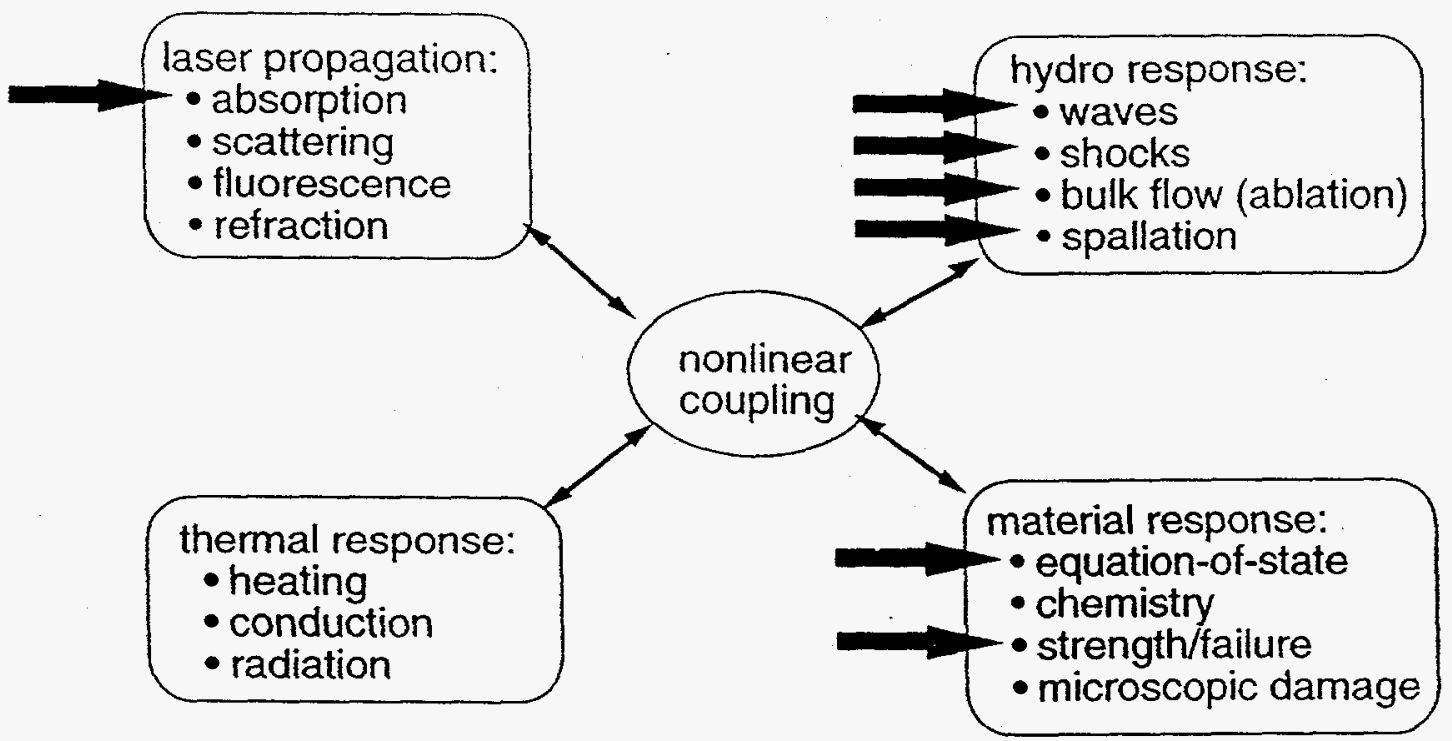

Figure 1. The LATIS computer program simulates laser-tissue interaction by considering 4 groups of coupled processes. The arrows indicate important processes which have recently been invoked for the present study.

The equations of state (EOS) needed for laser-tissue hydrodynamic simulations are 
generated in the form of tables and placed in a simple library format for easy access by LATIS and other hydrodynamic codes. The essential ingredients are pressure and internal energy as functions of temperature and density. The tables are produced by the Livermore EOS generator HQEOS6,7. This is a global model including solid, liquid, vapor, and plasma states over a wide range of temperatures and densities. The HQEOS model is based on thermodynamic functions composed of a sum of electronic, ion motion, and chemical binding terms. These terms are approximated by several simple physics models depending on the state of the material. The models have generally been found adequate for a wide range of applications. For the laser-tissue problem, we have generated EOS tables for tooth enamel (hydroxyapatite) by fitting the physics models to available experimental data. We take data for the bulk modulus and specific heat from refs. 8 and 9 . We estimate a cohesive energy from similar materials. We have made 2 EOSs with cohesive energies of 2 and $5 \times 10^{10} \mathrm{erg} / \mathrm{g}$ to test the sensitivity of the results to this parameter. The input data and therefore the EOS for enamel is still approximate at this time; future experimental measurements of the fundamental EOS parameters are desired to improve the accuracy.

The process of USPL ablation divides clearly into three distinct time periods: the laser, hydrodynamic, and thermal periods. Three linked calculations can be done to model the physical processes occurring during each time period.

The first time period is that of laser irradiation. The propagation and absorption of the laser light occur nearly instantaneously during the time the laser is on, typically 300 fsec. During this period, very little hydrodynamic motion nor heat conduction take place. Because the focal spot is typically much larger than the absorption depth, 1-D planar geometry can be assumed. A wave description of the laser light is necessary (as compared to the usual intensity description used in tissue optics) since the absorption occurs in an evanescent wave in the self-generated plasma at the tissue surface. A simple model for the formation of the plasma via multi-photon and collisional ionization is given in ref. 10. This model has been used in an approximate WKB solution of the wave equation absorption in ref. 10.

The second time period covers the ablation hydrodynamics. The characteristic time scale for this process is the deposition depth divided by the sound speed, approximately $10^{-5} \mathrm{~cm} / 3 \times 10^{5} \mathrm{~cm} / \mathrm{s} \approx 30 \mathrm{psec}$. Given the energy deposition as an initial condition,we simulate the hydrodynamic motion in time. The program solves the partial differential equations (first order in time, second order in space) describing mass, momentum and energy conservation via Lagrangian finite difference methods. In the Lagrangian method, finite spatial zones define small mass volumes which move with the material. The time step is actively controlled both by stability criteria (e. g. the "Courant limit") as well as by accuracy criteria on the various variables such as density and temperature. The solution produces space-time variations of the velocity, temperature, density, and pressure of the material. From these quantities we calculate the ablation depth and ablation efficiency, the thermal energy left in the tooth (the "thermal residual"), and mechanical coupling to the remaining tissue.

The third period of interest is that over which heat is transported to the center of the 
tooth, where we are concerned with thermal damage to the pulp. This timescale is approximately the square of the distance to the center of the tooth divided by the thermal diffusivity, of order $(0.1 \mathrm{~cm})^{2} /\left(5 \times 10^{-3} \mathrm{~cm}^{2} / \mathrm{s}\right) \approx 2 \mathrm{sec}$. To model the heat transport during this period we do 2-D calculations, taking the residual energy from the hydro calculation as an initial condition.

\section{RESULTS OF HYDRODYNAMIC STMULATIONS}

The laser irradiation geometry and the numerical zoning used in the hydro simulations are shown in Figure 2. The sufficiency of 1-D planar geometry is illustrated by the large ratio of laser focal spot diameter to the absorption and ablation depths. The zoning is feathered towards the surface of the tooth in order to resolve the laser deposition region while avoiding the use an extremely large number of zones to cover the desired depth of $100 \mu \mathrm{m}$. For the current simulations, 80 zones were used.

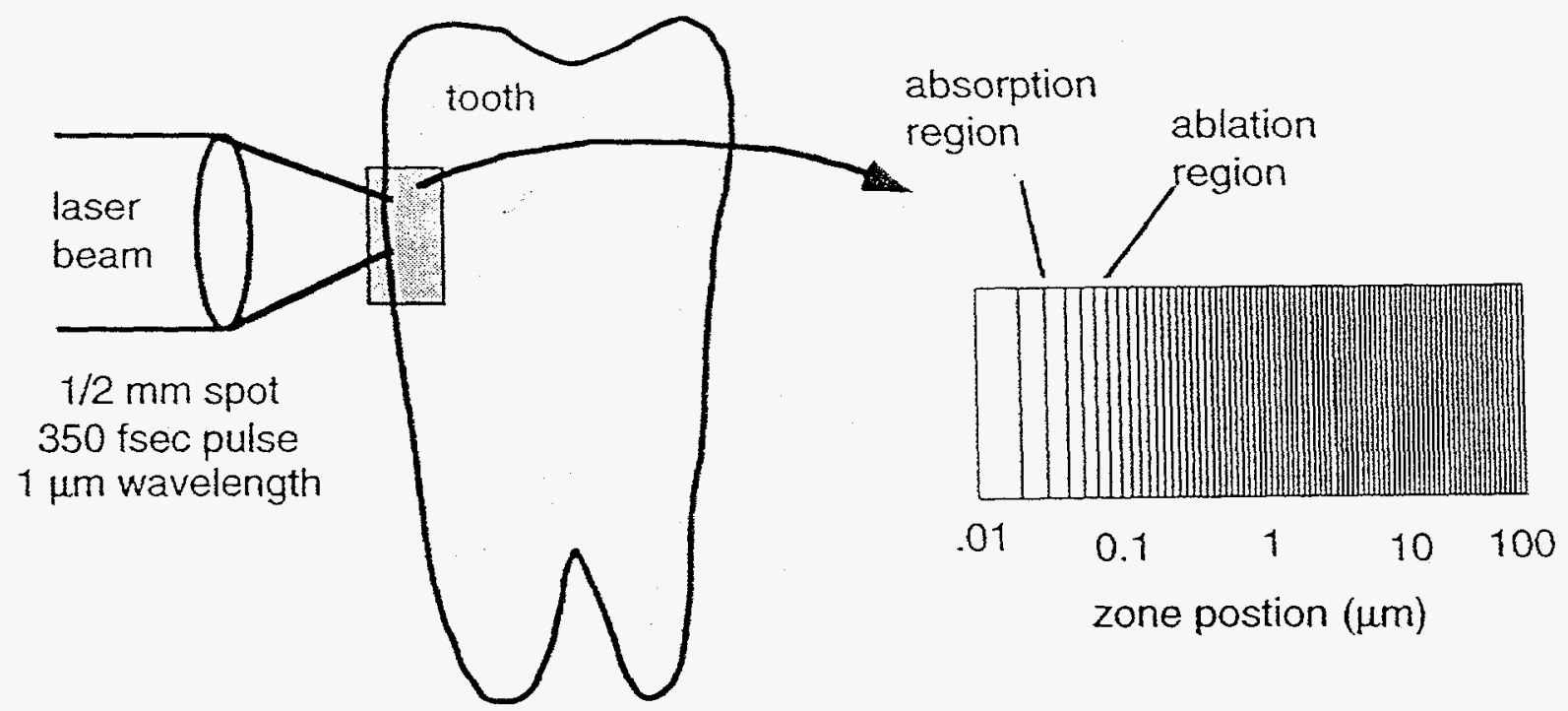

Figure 2. The interaction region of the laser with the tooth is modeled by a 1-D planar set of zones.

A set of hydro simulations have been done varying the laser energy fluence between 0.3 and $30 \mathrm{~J} / \mathrm{cm}^{2}$, corresponding to an energy between 2 and $20 \mathrm{~mJ}$ for a $1 / 2 \mathrm{~mm}$ diameter spot, as used in recent experiments at LLNL $^{1}$. We assume an instantaneous, spatially exponential energy addition to the tissue, approximating the laser deposition. In the parameter study we assumed that $30 \%$ of the laser light is absorbed for all cases. The absorption depth was varied between 0.03 and $1 \mu \mathrm{m}$, as indicated from 1-D WKB calculations ${ }^{10}$.

To illustrate the results and interpretation of the simulations, we show details for a "standard" run with incidence fluence $=9 \mathrm{~J} / \mathrm{cm}^{2}$ and absorption depth $=0.1 \mu \mathrm{m}$.

Figure 3 shows the calculated time variation of temperature of selected Lagrangian zones. At early times (less than about $1 \mathrm{psec}$ ) the temperatures directly reflect the 
deposited energy. The initial temperature is $6 \times 10^{4}{ }^{\circ} \mathrm{C}$ at the surface, and decreases approximately exponentially with depth. The temperature is not strictly exponential is due to a slight variation of the heat capacity. The decrease of the temperature of the outer zones (initial position $<.18 \mu \mathrm{m}$ ) with time, beginning at 1 to $30 \mathrm{psec}$, is caused by hydrodynamic expansion cooling. The ablating zones (those with initial position $<.32$ $\mu \mathrm{m})$ can be identified by their asymptotic cooling at long times. The remaining zones are identified by their constant temperatures at late times ( $10 \mathrm{nsec})$. Another feature evident in Figure 3 is the shock heating of the lower zones, occurring at times between 20 and 100 psec. The shock wave is driven into the tooth by the high pressures initially created in the laser absorption region. For zones near the ablation depth e.g. the zone with initial position $=.30 \mu \mathrm{m}$, the shock heating adds to the initial laser heating to give that material sufficient temperature to ablate, whereas for lower zones (e.g. that with initial position $=0.58 \mu \mathrm{m}$ ) the shock heating is not sufficient to cause ablation.

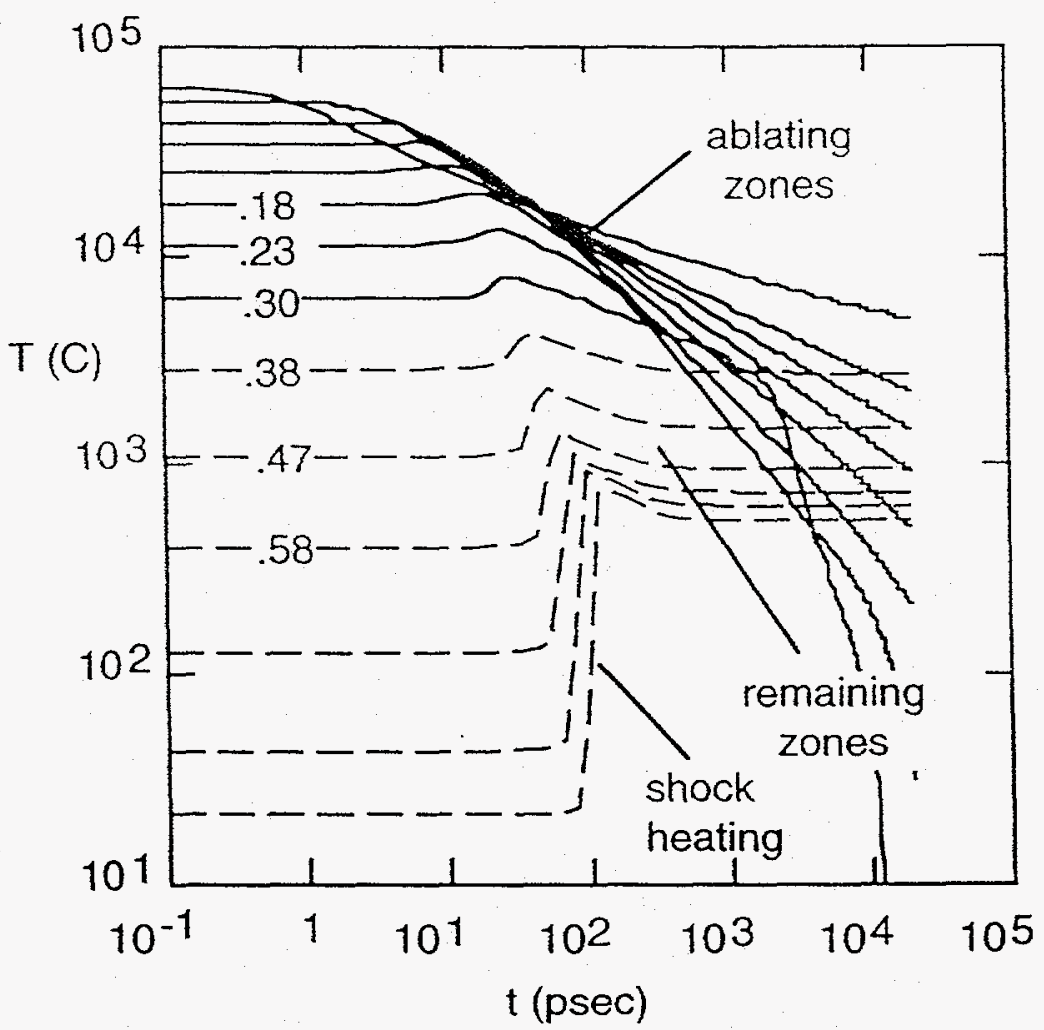

Figure 3. The time history of temperature for various zones illustrates the initial heating, the shock heating, and the division between ablating and remaining material. The temperatures of every other zone between zone 1 and zone 27 are shown. The initial positions (in $\mu \mathrm{m}$ ) of selected zones are indicated at the left. The ablating zones are solid, while remaining zones are dashed. The incident fluence is $9 \mathrm{~J} / \mathrm{cm}^{2}$ and the absorption depth is $0.1 \mu \mathrm{m}$

Another view of the laser-interaction process is illustrated by plots of density and pressure vs. position given in Figures $4 \mathrm{a}$ and $4 \mathrm{~b}$. The density plot illustrates both the ablation of the outer material, as its density drops with time up to about 300 psec, and the stress wave launched into the remaining tissue. The final ablation depth is indicated by 
the sharp transition between low and high density at late time. In this example the ablation depth is $0.32 \mu \mathrm{m}$. The pressure plot clearly shows the stress wave launched into the tooth. This wave moves at a rate of approximately $5 \mu \mathrm{m} / \mathrm{nsec}$, somewhat faster than the room temperature sound speed. It drops in peak pressure, and broadens due to the shock dissipation and velocity dispersion. Peak pressures at $50 \mu \mathrm{m}$ depth are predicted to be approximately $10 \mathrm{kbar}$. Relatively small (approximately 1 kbar) negative pressures (i. e. tensile stresses) develop after the positive pressure pulse passes at times around $5 \mathrm{nsec}$. These stresses, both positive and negative may damage the tissue, and lead to extra material removal via spallation. Accurate determinations of the damage thresholds for such short stress pulses are necessary to assess the degree and nature of such damage.
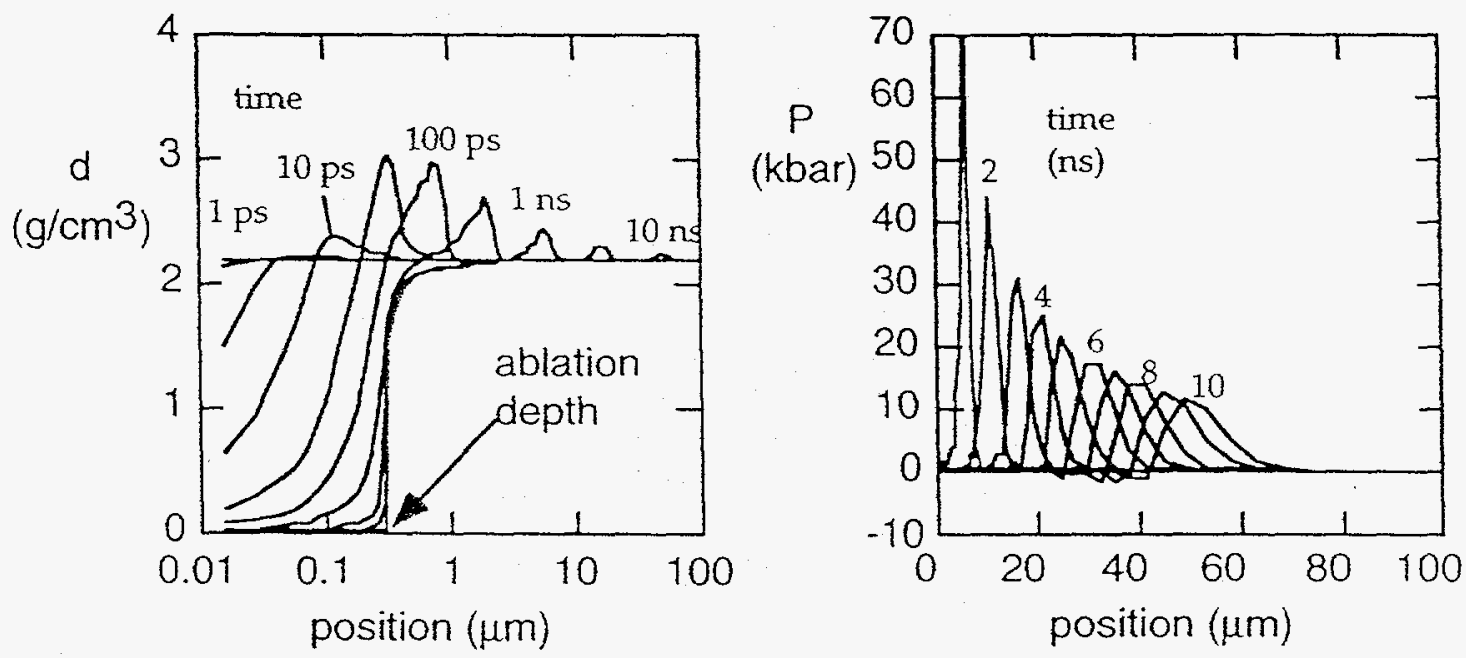

Figure 4. The ablation of material and the mechanical coupling into the tooth are shown in these plots of density (a) and pressure (b) versus initial position. Every 2nd curve is labeled by time. The density and pressure are shown on different space and time scales to illustrate different effects. The incident fluence is $9 \mathrm{~J} / \mathrm{cm}^{2}$ and the absorption depth is $0.1 \mu \mathrm{m}$.

The primary result of a hydro simulation is the ablation depth. This can be characterized by the ablation efficiency defined as the depth of material removed per unit of energy fluence delivered. The ablation efficiencies resulting from a variation of the incident fluence are given in Figure 5. Also shown in Figure 5 are experimentally measured ablation efficiencies using $350 \mathrm{fsec}$ pulses of $1 \mu \mathrm{m}$ light ${ }^{1}$. Note that the experimental scale for ablation efficiency is 3 times that of the simulations. Both results show the same trends, namely a sharp rise from a threshold fluence, which is $0.3 \mathrm{~J} / \mathrm{cm}^{2}$ in the model and $0.75 \mathrm{~J} / \mathrm{cm}^{2}$ in the experiment, a peak at 1 to $3 \mathrm{~J} / \mathrm{cm}^{2}$ followed by a drop at higher fluence. In the simulations the threshold is explained by the existence of a threshold temperature to which material must be heated in order to ablate (see section 4). In reality (i.e. experiment), an absorption threshold effect is also expected, due to the highly nonlinear nature of the multi-photon ionization initiated ionization cascade process ${ }^{10}$. The decrease of efficiency at high fluence is understood as an overheating effect of the surface layers. Because of the fixed exponential absorption function, extra energy goes mainly into the surface layers but not into deeper layers. Thus very little 
extra material is ablated at higher fluences. In reality, the surface layers are expected to become increasingly reflective of the laser light at higher intensities, leading to a further reduction of efficiency, not included in our models. Optimal ablation efficiencies are predicted to be about $\left.0.1 \mu \mathrm{m} /\left[\mathrm{J} / \mathrm{cm}^{2}\right)\right]$ for the parameters of the model, while the peak measured efficiencies are approximately $\left.0.3 \mu \mathrm{m} /\left[\mathrm{J} / \mathrm{cm}^{2}\right)\right]$.

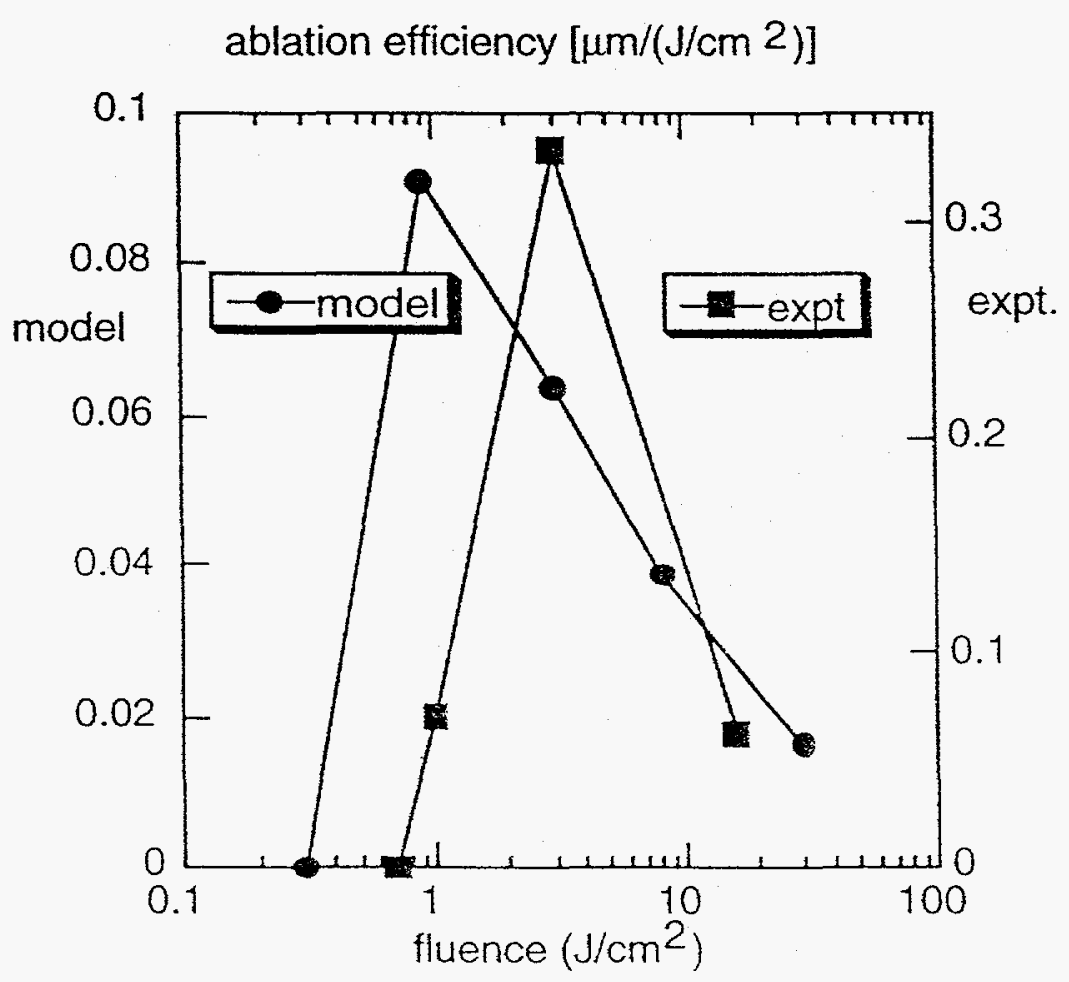

Figure 5. Ablation efficiency vs. fluence. The model assumes an absorption depth of $0.1 \mu \mathrm{m}$ and an absorption fraction of $30 \%$. Experimental data is from ref. 1 .

We have studied the effect of varying other input data and parameters of the model, as indicated in Table 1. The results are all for an incident fluence of $9 \mathrm{~J} / \mathrm{cm}^{2}$. At this fluence we predict an increase in efficiency with increasing absorption depth. This results from the fact that the temperatures achieved in the outer regions are all above the critical value necessary for ablation for the absorption depths studied (see section 4). Thus a larger absorption depth produces a large ablation depth. If the absorption depth were increased still further a value would be reached at which the surface temperature drops below the critical value and the ablation would be halted. We estimate that this would occur at $2.4 \mu \mathrm{m}$ for a fluence, $F=9 \mathrm{~J} / \mathrm{cm}^{2}$. We have also done a simulation with a weaker equation-of-state having a cohesive energy of $2 \times 10^{10} \mathrm{erg} / \mathrm{g}$ rather than $5 \times 10^{10}$ $\mathrm{erg} / \mathrm{g}$ for the standard case. This allows material at lower temperatures to ablate and therefore results in the removal of more material. This variation points out the need for a better determination of the EOS parameters, specifically the cohesive energy.

Another simulation was done including a preliminary material failure model. In this model, we assumed certain compressive and tensile yield strengths, namely $3 \mathrm{kbar}$ 
for compression and $1 \mathrm{kbar}$ for tension, approximate values from refs. 8 and 9 . We limit tensile stresses to be always less than the tensile strength value. In addition, if the compressive stress in a zone exceeds the compressive strength, we assume that the material strength is then compromised and thereafter set the tensile strength to zero in that zone. This process represents a "crushing" of the material resulting in a great weakening of its strength. We find that a large region $(\sim 70 \mu \mathrm{m})$ is ultimately subjected to compressive strengths larger than $3 \mathrm{kbar}$ and thus then ultimately ablates due to this material failure mechanism. However, the value of compressive yield strength quoted in the literature is very uncertain, and is much lower than similar insulating materials such as glass (yield strength $=70 \mathrm{kbar}$ ). Additionally, the quoted yields are for static stresses, whereas we may expect very different yield values for the short duration stresses experienced in the USPL case. The simulation results show stress levels considerably larger than the static yield strengths in the literature, but the experiments clearly show less damage. Thus the results of the material failure calculations, although very interesting, must be regarded as very preliminary at this time.

\section{Table 1. Parameter variations (at $F=9 \mathrm{~J} / \mathrm{cm}^{2}$ )}

\begin{tabular}{lll} 
case & $\begin{array}{l}\text { abs. depth } \\
(\mu \mathrm{m})\end{array}$ & $\begin{array}{l}\text { ablation efficiency } \\
{\left[\mu \mathrm{m} /\left(\mathrm{J} / \mathrm{cm}^{2}\right)\right]}\end{array}$ \\
\hdashline experiment & 1 & 0.1 \\
small depth & .03 & .01 \\
standard model & .1 & .03 \\
large depth & .3 & .05 \\
"weak" EOS & .1 & .04 \\
material failure & .1 & $(>.1)$
\end{tabular}

\section{ANALYTIC ABLATION MODEL}

In order to ablate via the hydrodynamic process considered in section 4 , material must be heated enough to flow smoothly from the solid phase to the vapor phase. It must overcome the so-called "vapor dome," or otherwise it will experience a restoring force due to negative pressures. The necessary conditions for ablation of a given parcel of material can be understood by considering EOS adiabats (curves of constant entropy) in the pressure-volume (P-V) plane, as illustrated by the solid curves in Figure 6 . These curves are calculated strictly from the EOS tables; not from a hydro simulation. Each adiabat is labeled by initial temperature reached upon heating at constant volume $(=0.45$ $\mathrm{cm}^{3} / \mathrm{g}$ ), as assumed in the hydro models discussed in section 4 . Even though the curves are labeled by a temperature, they are not isotherms. We consider adiabats since we expect each parcel of material (represented by a Lagrangian zone in the hydro calculations) to conserve entropy (equal to the sum of internal energy plus PdV work) after the initial heating because heat conduction is very slow on the hydro timescale. Shock heating adds some entropy; however it is generally small in our runs. The 
analysis can be easily extended to consider shock heating followed by adiabatic flow. In Figure 6, we see that the higher adiabats go monotonically towards zero pressure at large volume, while the lower adiabats cross into the negative pressure region and then approach zero pressure from below. The critical adiabat is defined as that which divides the two cases, namely the lowest adiabat which does not pass into the negative pressure region. The initial temperature for the critical adiabat for our EOS, which we define as the "ablation temperature", i

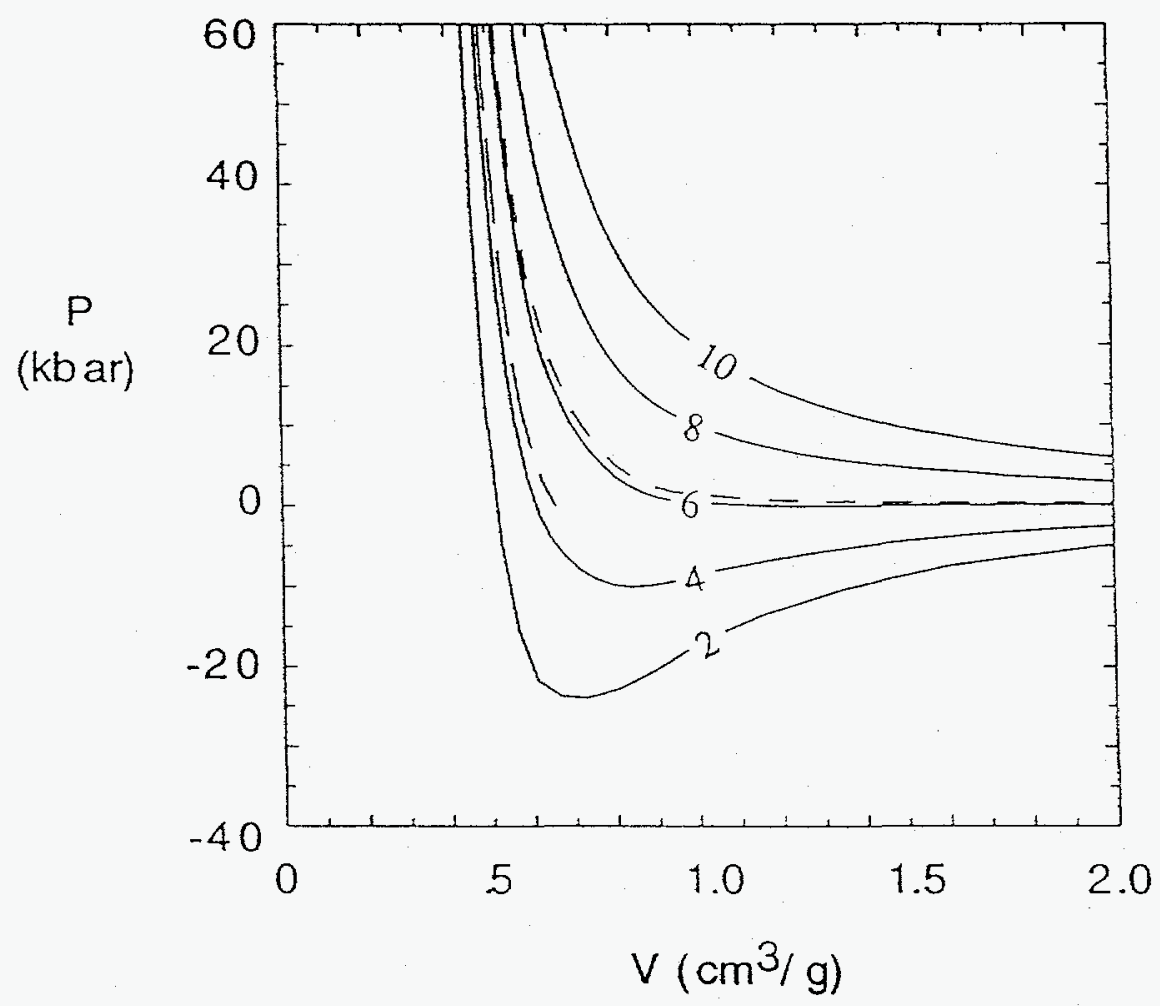

Figure 6 Solid lines are EOS adiabats, labeled by initial temperature $\left(10^{3}{ }^{\circ} \mathrm{C}\right)$ after heating at a constant volume $\left(=.45 \mathrm{~cm}^{3} / \mathrm{g}\right)$. The critical adiabat has initial temperature $\approx 6,000^{\circ} \mathrm{C}$. The dashed lines are P-V curves from two adjacent zones in the standard hydro simulation.

Relating the adiabats to ablation, we propose the hypothesis that for any given parcel of material to ablate, it must be heated above the critical adiabat, i.e. above a temperature of about $6,000 \mathrm{C}$, if the heating occurs instantaneously at constant volume. This hypothesis has been tested by considering the actual P-V trajectories of various zones near the ablation depth of our standard hydro simulation. Two of these trajectories are drawn in Figure 6. The long-dashed line is for a zone with initial position $=.36 \mu \mathrm{m}$ which does not ablate, whereas the short-dashed line is for the adjacent zone with initial position $=0.32 \mu \mathrm{m}$, which does ablate. The P-V curve for the non-ablating zone is below the critical adiabat. It returns to low pressure at a relatively small volume. The P-V curve for the ablating zone slightly exceeds the critical adiabat; it goes to very large volume as it approaches zero pressure. The different P-V behavior 
of these two zones is in agreement with our hypothesis that material must be heated to a super-critical adiabat to ablate. The application of this hypothesis gives an estimate of the ablation threshold fluence, $F_{t h}$, by determining when the outer most zones are just heated to the ablation temperature. This gives $F_{t h}=0.4 \mathrm{~J} / \mathrm{cm}^{2}$ for absorption depth, $z_{\mathrm{abs}}$, $=0.1 \mu \mathrm{m}$, in fairly good agreement with the hydro simulations. For fluences above this value the ablation depth is estimated as the depth at which the initial temperature drops below the ablation temperature, $\mathrm{z}_{\mathrm{abl}}=\mathrm{z}_{\mathrm{abs}} \ln \left(\mathrm{F} / \mathrm{F}_{\mathrm{th}}\right)$. This represents a slight underestimate of the ablation depth since the shock wave will provide additional heating.

\section{HEAT CONDUCTION SIMULATIONS}

Each laser pulse leaves a certain residual energy in the tooth. At the high repetition rates necessary for high-speed drilling (e.g. $500-1,000 \mathrm{~Hz}$ ), this heat will build-up and may damage the pulp in the interior of the tooth. To model such an effect we have taken the residual energy from the single pulse hydro simulation and multiplied it by 500 to represent 500 pulses. We then simulate the heat conduction in a 2 $D$ geometry to see how far the heat will penetrate into the tooth. Results for the 2-D temperature distribution at $1 \mathrm{sec}$ are given in Figure 7 . We have assumed a simple geometry with 3 layers-enamel, dentin and pulp as indicated in a cylinder of radius 2.5 $\mathrm{cm}$. The temperature at a depth of $0.1 \mathrm{~cm}$ (approximately the enamel-dentin boundary) is approximately $18^{\circ} \mathrm{C}$, while it is less than $1^{\circ} \mathrm{C}$ at $0.4 \mathrm{~cm}$ the dentin-pulp boundary. This calculation illustrates the capability to couple the hydro simulations to long-time conduction simulations and it indicates that extensive heating of the pulp may be avoided with USPL drilling.

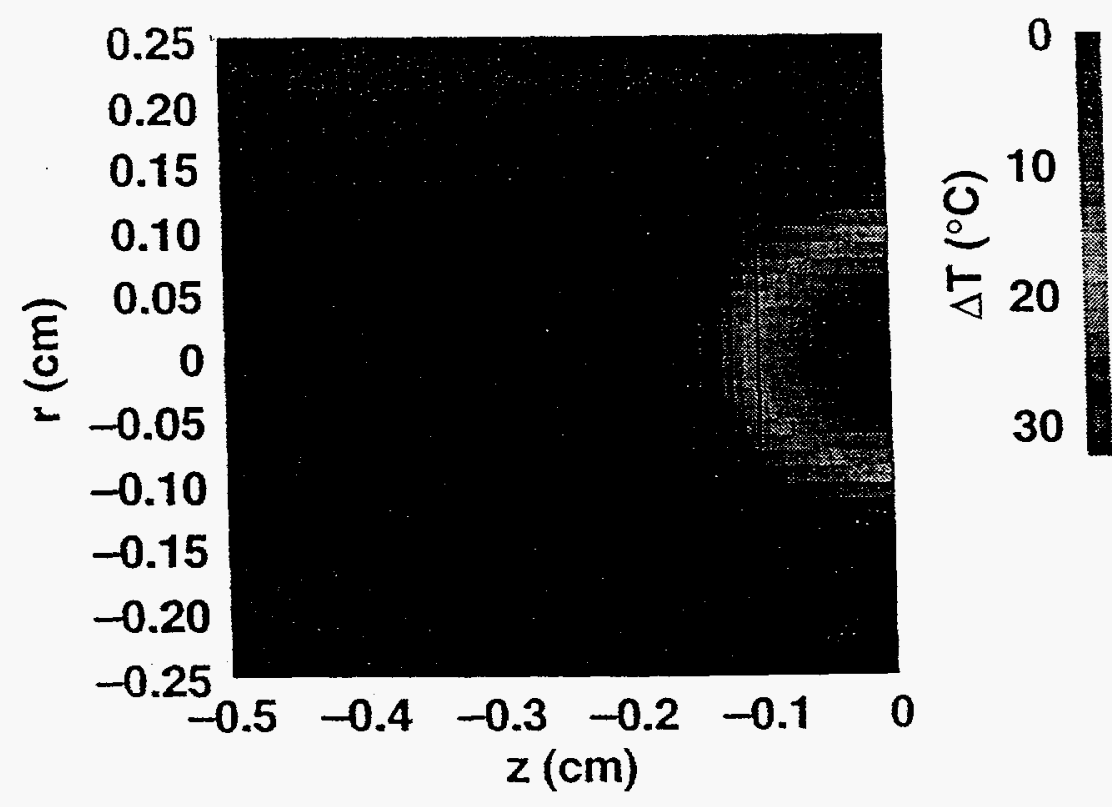

Figure 7 Temperature distribution at $1 \mathrm{sec}$ after 500 pulses. 


\section{FUTURE WORK AND CONCLUSIONS}

Several enhancements to our model are being developed or have been identified for future work. We are developing a laser propagation and absorption package incorporated in the LATIS code based on a 1-D electromagnetic wave solver ${ }^{11}$. This will produce self-consistent absorption profiles and absorption fractions as the incidence fluence is varied. We are also improving the material failure model which will allow better simulations of effects such as spallation of material by negative pressures, as well as compressive, tensile and shear stress induced tissue damage. We are looking at rate models for the yield parameters, as well as other stress limits. We also plan to improve the EOS for hard tissues to better model the ablation process. In order to improve the material failure model and the EOS, experimental data, such as the heat and temperature of vaporization, temperature dependent heat capacity and conductivity, and better values for time-dependent material strengths are needed. In addition to hydrodynamic ablation considered in section 4 , material may be removed by evaporation at temperatures below the critical point. In this case, the material undergoes a phase transition between liquid or solid and vapor which occurs at a rate which depends on the equilibrium vapor pressure. This is fundamentally a non-equilibrium processes and may be treated by a rate equation and a 2-phase EOS. Such calculations will be considered in future work. Finally, refinements to the thermal calculations are being pursued, including the development and application of a 3-D computer program which will let us more accurately represent the whole tooth.

In this paper we have demonstrated the role of numerical simulations in studying ultra-short-pulse dental drilling. We have identified the fundamental mechanisms involved in USPL laser-hard tissue interaction. Absorption proceeds by a multi-photon initiated plasma; ablation involves supercritical heating of the material. We have illustrated mechanical coupling to the remaining material via a propagation shock wave, and have studied thermal coupling via sub-critical and shock heating. We have shown a calculation of the thermal conduction over long time periods. Several desired refinements to the model have been identified. We believe that numerical simulation will be a valuable tool in designing and analyzing systems for ultra-short-pulse laser tissue ablation.

\section{ACKNOWLEDGEMENTS}

This work was performed under the auspices of the U. S. Department of Energy by the Lawrence Livermore National Laboratory under contract number W-7405-ENG-48.

\section{REFERENCES}

1. J. Neev, A. M. Rubenchik, L. B. Da Silva, B. C. Stuart, M. D. Feit, P. Wilder-Smith, D.

L. Matthews, and M. D. Perry, "Dentin and enamel ablation with ultra-short pulse lasers: considerations for a practical drilling device," to appear in Applied Optics (1996).

2. M. D. Perry and B. C. Stuart, "Development of ultra-short pulse laser systems for 
hard tissue processing," in Lasers in Dentistry II, H. A. Widgdor, J. D. Featherstone, J. M. White, and J. Neev, Eds., Proc. SPIE 2672 (1996, in press)

3. R. A. London, M. E. Glinsky, G. B. Zimmerman, D. C. Eder, and S. L. Jacques, "Coupled light transport-heat diffusion model for laser dosimetry with dynamic optical properties," in Laser Tissue Interaction VI, S. L. Jacques, Ed., Proc. SPIE 2391 (1995), p. 431.

4. M. E. Glinsky, R. A. London, G. B. Zimmerman, and S. L. Jacques, "Modeling of endovascular patch welding using the computer program LATIS," in Laser Tissue Interaction VI, S. L. Jacques, Ed., Proc. SPIE 2391 (1995), p. 262.

5. G. B. Zimmerman and W. L. Kruer, "Numerical simulation of laser initiated fusion," Commun. Plasma Phys. Controlled Fusion, 11, 82 (1975).

6. R. M. More, K. H. Warren, D. A. Young and G. B. Zimmerman, "A new quotidian equation of state (QEOS) for hot dense matter," Phys. Fluids, 31, 3059 (1988).

7. D. A. Young and E. M. Corey, "A new global equation of state model for hot, dense matter," J. Appl. Phys. 78, 3748 (1995).

8. F. A. Duck, Physical properties of tissue (San Diego; Academic),(1990).

9. J. B. Park, Biomaterials science and engineèring (New York: Plenum Press), 1984.

10. M. D. Feit, A. M. Rubenchik, and B. W. Shore, "Unique aspects of energy deposition in the fs pulse regime," in Lasers in Dentistry II, H. A. Widgdor, J. D. Featherstone, J. M. White, and J. Neev, Eds., Proc. SPIE 2672 (1996, in press)

11. W. E. Alley, "A Maxwell equation solver for the simulation of moderately intense ultra-short pulse laser experiments," (Univ. of California Lawrence Livermore National Laboratory report UCRL-LR 105820-92 (1992), p. 160. 


\section{SPIE CODE NUMBER: 2681-32}

Dr. Richard A. London

Lawrence Livermore National Lab.

Laser Target Design Div.

$\mathrm{L}-477$

7000 East Ave

Livermore, CA 94550

Dear Dr. London:

It is our pleasure to confirm the acceptance of your abstract for the conference Laser-Tissue Interaction VII, part of SPIE's BiOS '96, 27 January-2 February 1996, San Jose, CA. Your paper on Computational modeling of ultrashort-pulse ablation of enamel is scheduled for 30-Jan-1996. Please consult the Advance Technical Program, which will be available and mailed in October, for approximate time of participation. The Advance Technical Program will also contain the following information:

- hotel accommodations form

- registration form (authors pay reduced fees that include proceedings and hosted events)

- complete list of technical presentations and special events.

Your paper has been scheduled for oral presentation*. Please see page 3 of the attached Author's Guide for Conferences and Manuscripts for pertinent details relating to this type of participation.

Please note that a 200-word Camera-Ready Abstract is due 25 November 1995 (see instructions in Guide, p. 10; mats and return envelope are included) and your manuscript is due 2 January 1996. It is most important that the manuscript due date be strictly observed, as our new publications policy requires proceedings volumes to be published shortly after the symposium. Late manuscripts run the risk of not being published.

If you have questions or problems with your manuscript or presentation, please contact SPIE.

Sincerely,

Marilyn E. Gorsuch

BiOS '96

Internet marilyn@spie.org; Phone: 360/676-3290; Fax: 360/647-1445

*Note: If poster presentation, prepare material for 4' by 4' board (see page 9).

Reminder! Important Due Dates

Camera-Ready Abstracts Due: 25 November 1995

Manuscripts Due: 2 January 1996 